\title{
Effectiveness of the Dader Method for Pharmaceutical Care on Patients with Bipolar I Disorder: Results from the EMDADER-TAB Study
}

\author{
Andrea Salazar-Ospina, PhD, PharmD; Pedro Amariles, PhD, PharmD; \\ Jaime A. Hincapié-García, BPharm, MSc; Sebastián González-Avendaño, MD; \\ Dora M. Benjumea, PhD, PharmD; Maria José Faus, PhD, PharmD; and Luis F. Rodriguez, MD
}

\begin{abstract}
BACKGROUND: Bipolar I disorder (BD-I) is a chronic illness characterized by relapses alternating with periods of remission. Pharmacists can contribute to improved health outcomes in these patients through pharmaceutical care in association with a multidisciplinary health team; however, more evidence derived from randomized controlled trials (RCTs) is needed to demonstrate the effect of pharmaceutical care on patients with BD-I.
\end{abstract}

OBJECTIVE: To assess the effectiveness of a pharmaceutical intervention using the Dader Method on patients with BD-I, measured by the decrease in the number of hospitalizations, emergency service consultations, and unscheduled outpatient visits from baseline through 1 year of follow-up.

METHODS: This study is based on the EMDADER-TAB trial, which was an RCT designed to compare pharmaceutical care with the usual care given to outpatients with BD-I in a psychiatric clinic. The main outcome was the use of health care services, using Kaplan-Meier methods and Cox regression. The trial protocol was registered in ClinicalTrials.gov (Identifier NCT01750255).

RESULTS: 92 patients were included in the EMDADER-TAB study: 43 pharmaceutical care patients (intervention group) and 49 usual care patients (control group). At baseline, no significant differences in demographic and clinical characteristics were found across the 2 groups. After 1 year of follow-up, the risk of hospitalizations and emergencies was higher for the control group than for the intervention group (HR=9.03, $P=0.042$; $\mathrm{HR}=3.38, P=0.034$, respectively); however, the risk of unscheduled outpatient visits was higher for the intervention group (HR=4.18, $P=0.028)$. There was no "placebo" treatment, and patients in the control group might have produced positive outcomes and reduced the magnitude of differences compared with the intervention group.

CONCLUSIONS: Compared with usual care, pharmaceutical care significantly reduced hospitalizations and emergency service consultations by outpatients with BD-I.

J Manag Care Spec Pharm. 2017;23(1):74-84

Copyright @ 2017, Academy of Managed Care Pharmacy. All rights reserved.

\section{What is already known about this subject}

Pharmacotherapy has traditionally been the cornerstone for managing bipolar disorder; however, despite having adequate drug therapy, a percentage of this population does not achieve clinical stability and continues to suffer multiple relapses.
Pharmacists provide pharmaceutical care to patients by detecting, preventing, and solving drug-related problems or negative clinical outcomes.

The Dader Method is a tool to help provide pharmaceutical care, so that pharmacists can achieve the most effective and safe pharmacotherapy as possible.

\section{What this study adds}

This study is based on the (EMDADER-TAB) trial, which was the first randomized controlled trial conducted in Colombia that assessed the effect of a pharmaceutical intervention using the Dader Method for patients with bipolar disorder I (BD-I), measured by the decrease in the use of health care services.

Pharmaceutical care based on the Dader Method significantly reduced hospitalizations and emergency service consultations, during 1 year of follow-up in patients with BD-I.

The EMDADER-TAB study demonstrates the positive effect of pharmacist involvement as part of a multidisciplinary mental health team in achieving therapeutic goals in patients with BD-I.

B ipolar disorder (BD) is a severe psychiatric disorder characterized by the presence of manic or hypomanic episodes and major depression, usually separated by asymptomatic intervals. BD-I patients have at least 1 manic episode and nearly always experience major depressive and hypomanic episodes throughout life. ${ }^{1}$ BD is currently ranked as the sixth leading cause of disability-adjusted life years worldwide among all diseases, and life expectancy is significantly reduced for those who suffer this condition. ${ }^{2} \mathrm{BD}$ involves men and women equally with a peak age onset typically between ages 18-20 years. ${ }^{3}$ In Colombia, the lifetime prevalence of BD-I is $1.8 \%{ }^{4}$ $\mathrm{BD}$ patients are more likely to have poor adherence, drug interactions, self-medication, drug-related side effects, and adverse reactions. ${ }^{5}$ Additionally, $\mathrm{BD}$ is comorbid with other disorders such as personality and eating disorders, suicide attempts, and current anxiety or substance use disorders. ${ }^{6}$ Moreover, the presence of $\mathrm{BD}$ with comorbid conditions is associated with a negative impact and increase in demand for health resources (hospitalizations, emergency room visits, and outpatient visits) 


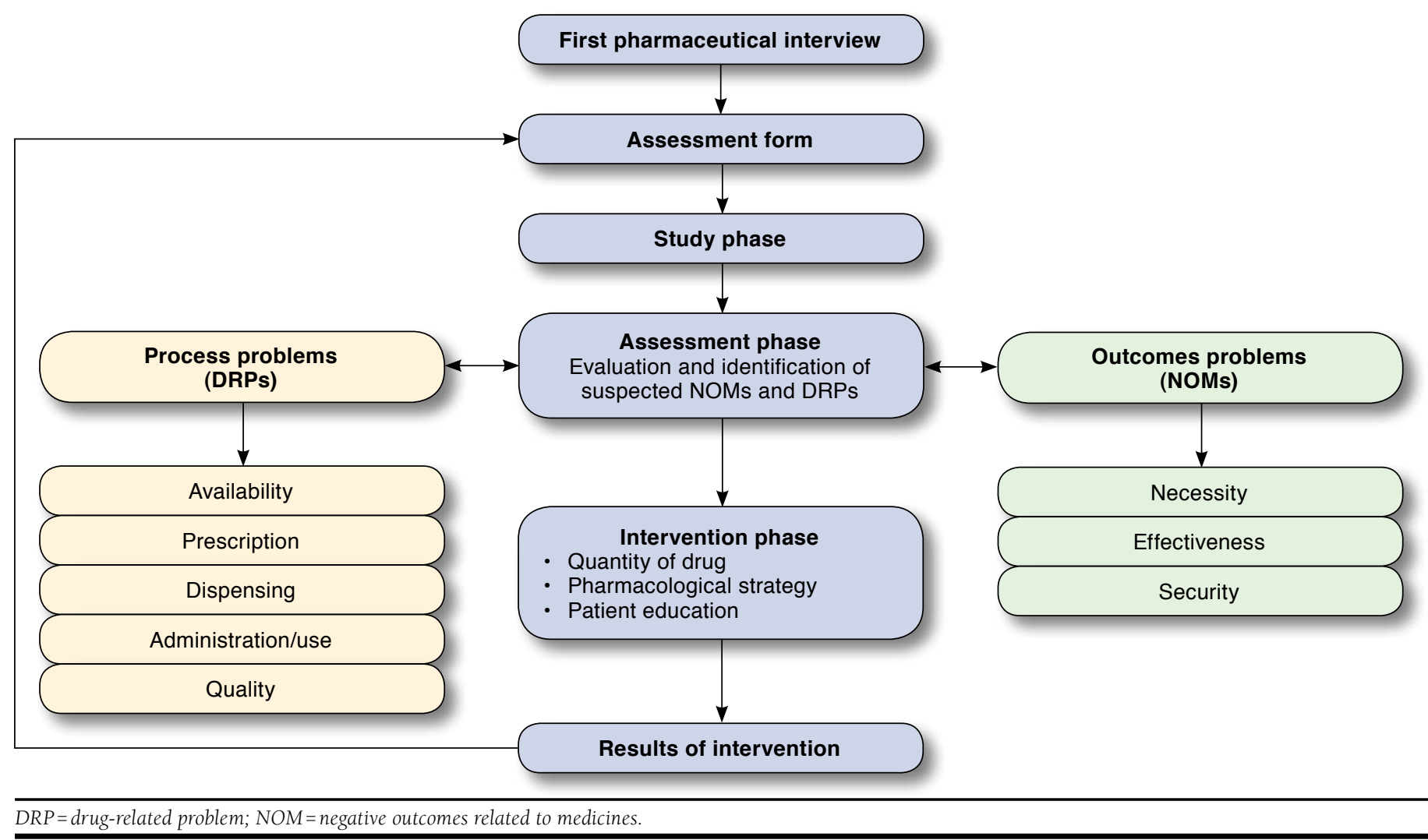

and indirect costs, such as work disability, unemployment, and loss of productivity. ${ }^{7,8}$

In the literature, there are reviews that highlight the role of pharmacists in achieving therapeutic goals and show that their inclusion on the health care team improves the appropriate use of medication and treatment adherence. ${ }^{9-11}$ Also, there are studies of patients with mental illness showing that pharmacists contribute to the achievement of therapeutic goals by providing pharmaceutical care. For example, it has been shown that pharmaceutical care for long-term schizophrenia inpatients improves medication adherence and treatment satisfaction. ${ }^{12}$ For depressed patients, the use of pharmaceutical care resulted in a reduction of depressive and anxiety symptoms. ${ }^{13}$

Professionally, pharmaceutical care refers to the responsibility that pharmacists have for patient medication needs. Pharmaceutical care is provided by means of detection, prevention, and solution of drug-related problems (DRPs) or a negative outcome related to medicine (NOM) and involves continuous attention on the part of the pharmacist and collaboration with patients and other health care professionals to reach concrete results that improve patient quality of life. ${ }^{14,15}$ The Dader Method is a tool to help provide pharmaceutical care so that pharmacists can achieve the most effective and safe pharmacotherapy as possible. According to the Dader Method, a pharmacist conducts a pharmaceutical interview, where drug information and patient health problems are recorded in order to create an assessment form. After the pharmaceutical interview, the pharmacist makes an assessment and identifies any DRPs and NOMs resulting from the patient's pharmacotherapy. Then, the pharmacist suggests any necessary interventions and verifies and reassesses the outcomes of the interventions. ${ }^{16-18}$ These interventions can address drug quantity (e.g., modifying dosage or frequency), pharmacological strategy (e.g., adding or changing any drug), and patient education. ${ }^{19}$ Figure 1 shows the Dader Method process.

Some studies have highlighted the role of the pharmacist in achieving therapeutic goals as part of a mental health team, but none of these studies demonstrated any benefits in clinically meaningful outcomes (e.g., hospital admissions, morbidity, or mortality); therefore, there is a need for the development of studies that provide a higher quality of evidence. ${ }^{2}$ Despite recent advances, there are no studies addressing pharmaceutical care in patients with BD that show the benefits that have been obtained from pharmaceutical care in other illnesses. 
More evidence is needed to demonstrate the effectiveness of pharmaceutical care in patients with BD-I.

The purpose of this study was to assess the effectiveness of a pharmaceutical intervention using the Dader Method on patients with BD-I, as measured by the decrease in the number of hospitalizations, emergency service consultations, and unscheduled outpatient visits from baseline to 1 year of follow-up, compared with usual care received by patients in a psychiatric clinic.

\section{Methods}

\section{Participants and Setting}

This study is based on the EMDADER-TAB trial, which was a randomized controlled trial (RCT) designed to compare the effectiveness of a pharmaceutical intervention using the Dader Method with usual care received by outpatients with BD-I in a psychiatric clinic in La Ceja, Antioquia, Colombia. The study began in November 2011 and ended in June 2014. Before the trial, the role of the pharmacist in the psychiatric clinic was mainly administrative. When the EMDADER-TAB trial began, a mental health pharmacist was commissioned to conduct clinical activities for the study. Detailed methods of the EMDADERTAB trial have been previously published, and the trial has been registered at ClinicalTrials.gov (identifier NCT0175025). ${ }^{21}$

\section{Participant Selection and Assignment}

Patients diagnosed with BD-I who were aged between 18 and 65 years and who had been discharged or referred from the outpatient service of the clinic were included in the study. The demographic characteristics of the population are shown in Table 1. Patients with the following were excluded from the study:

- First manic episode, schizoaffective disorder, BD-II, cyclothymia, and other bipolar spectrum disorders; personality disorders that seem like BD; and sociopathic disorder.

- Epilepsy; human immunodeficiency virus; and chronic decompensated disease (i.e., blood pressure values above 180/110 mmHg, total cholesterol above $300 \mathrm{mg} / \mathrm{dL}$, lowdensity cholesterol greater than $160 \mathrm{~mm} / \mathrm{dL}$, hemoglobin Alc greater than 9\%, and oxygen saturation lower than 90\%).

- Unable to comply with the protocol requirements because of severe alcohol and drug use.

- Pregnancy or breastfeeding.

- Intellectual disability, presence of any cognitive impairment impeding the comprehension and signing of the informed consent form, or illiteracy.

Two methods were used to recruit patients: (1) the pharmacist identified potential candidates for the study using patients' medical histories, and (2) psychiatrists identified potential participants who met the inclusion criteria. ${ }^{21}$ Once the inclusion and exclusion criteria were verified, the pharmacist asked patients for authorization to participate. If the patients agreed to participate, they were asked to sign the study's consent form. Recruitment time had a length of 30 months. Participants were randomized to intervention or control groups in sequential order, and they were followed for 12 months, starting from the date of their recruitment. Variables related to the primary outcomes were collected at baseline and every 3 months to complete the study year. With information from both groups, the outcomes were evaluated in terms of the rate of change from baseline to 1 year. According to the study protocol, clinic visits were scheduled at 3, 6, 9, and 12 months. Patients in both groups received phone calls from the pharmacist to encourage protocol compliance. The study patients were not blinded to treatment assignment, and both groups were seen by the same pharmacist.

\section{Intervention Group: Pharmaceutical Care}

Patients assigned to the intervention group received usual care, verbal and written counseling regarding $\mathrm{BD}$, and pharmaceutical care for 1 year from a specially trained pharmacist using the Dader Method. The pharmacist was experienced in the pharmaceutical care of psychiatric patients and had been trained by psychiatrists in the support and clinical management of the mentally ill.

During the year of follow-up, the intervention group received a weekly telephone call from the pharmacist until the end of the study as part of the pharmaceutical care program. The purpose of these calls was the gathering of information related to patient treatment adherence and effectiveness and pharmacotherapy safety. During these calls, the pharmacist did the following: (a) carried out a clinical evaluation assessing changes in mood, behavior, pattern of regular eating and sleeping, language, and thought; (b) emphasized the importance of patient education and the recognition and management of prodromal symptoms; (c) explained the proper use of bipolar drugs; (d) promoted therapeutic adherence; and (e) promoted healthy eating and lifestyle habits. After collecting the patient information, the pharmacist completed the assessment form and worked with the medical team, patient, and family/caregiver regarding any necessary interventions. After interventions were completed, the pharmacist evaluated and verified the results. The weekly calls allowed the pharmacist to monitor, support, and provide ongoing care to the participating patients. Through these calls, the intervention group was in constant contact with the pharmacist in order to identify, prevent, or promptly resolve problems related with the process and outcomes of pharmacotherapy.

\section{Control Group: Usual Care}

The control group received usual care, which consisted of routine dispensing and verbal and written counseling regarding BD. The written material contained mental health and BD information, which focused on the importance of adherence 


\section{Effectiveness of the Dader Method for Pharmaceutical Care on Patients with Bipolar I Disorder: Results from the EMDADER-TAB Study}

\section{TABLE 1 Demographic and Clinical Characteristics Related to Primary Goal at Baseline}

\begin{tabular}{|c|c|c|c|c|c|c|c|}
\hline \multirow{2}{*}{$\begin{array}{l}\text { Demographic and Clincal Variables } \\
\text { Age, mean }[S D]\end{array}$} & \multicolumn{2}{|c|}{$\begin{array}{l}\text { Total } \\
\mathrm{N}=92 \\
\mathrm{n}(\%)\end{array}$} & \multicolumn{2}{|c|}{$\begin{array}{c}\text { Control Group } \\
\mathrm{n}=49 \\
\mathrm{n}(\%) \\
\end{array}$} & \multicolumn{2}{|c|}{$\begin{array}{l}\text { Intervention Group } \\
\quad \mathrm{n}=43 \\
\mathrm{n}(\%)\end{array}$} & \multirow{2}{*}{$\begin{array}{r}P \text { Value } \\
0.448 \\
\end{array}$} \\
\hline & 42.8 & {$[10.9]$} & 43.7 & {$[11.8]$} & 41.9 & {$[9.9]$} & \\
\hline \multicolumn{8}{|l|}{ Gender } \\
\hline Female & 52 & $(56.5)$ & 29 & $(59.2)$ & 23 & $(53.5)$ & 0.582 \\
\hline \multicolumn{8}{|l|}{ Education } \\
\hline Elementary school or less & 32 & $(34.8)$ & 16 & $(32.7)$ & 16 & $(37.2)$ & \multirow{3}{*}{0.973} \\
\hline High school & 35 & $(38.0)$ & 19 & $(38.8)$ & 16 & $(37.2)$ & \\
\hline University education & 25 & $(27.2)$ & 14 & $(28.6)$ & 11 & $(25.6)$ & \\
\hline \multicolumn{8}{|l|}{ Marital status } \\
\hline Single & 23 & $(25.0)$ & 14 & $(28.6)$ & 9 & $(20.9)$ & \multirow{2}{*}{0.262} \\
\hline Married, common-law union & 52 & $(56.5)$ & 26 & $(53.1)$ & 26 & $(60.5)$ & \\
\hline \multicolumn{8}{|l|}{ Nuclear family } \\
\hline Family of origin & 34 & $(36.9)$ & 17 & $(34.7)$ & 17 & $(39.5)$ & \multirow{2}{*}{0.249} \\
\hline Own family & 55 & $(59.8)$ & 29 & $(59.2)$ & 26 & $(60.5)$ & \\
\hline \multicolumn{8}{|l|}{ Origin } \\
\hline Urban area & 72 & $(78.3)$ & 40 & $(81.6)$ & 32 & $(74.4)$ & 0.403 \\
\hline \multicolumn{8}{|l|}{ Occupation } \\
\hline Employee & 46 & $(50.0)$ & 21 & $(42.9)$ & 25 & $(58.2)$ & 0.365 \\
\hline \multicolumn{8}{|l|}{ Duration of illness, mean [SD], years } \\
\hline $1-5$ & 17 & {$[18.5]$} & 11 & {$[22.4]$} & 6 & {$[14.0]$} & \multirow{3}{*}{0.461} \\
\hline $6-10$ & 20 & {$[21.7]$} & 12 & {$[24.5]$} & 8 & {$[18.6]$} & \\
\hline$>10$ & 49 & {$[53.3]$} & 24 & {$[49.0]$} & 25 & {$[58.1]$} & \\
\hline Age of illness onset, years, mean [SD] & 29.5 & {$[10.5]$} & 29.9 & {$[11.8]$} & 28.9 & {$[8.9]$} & 0.805 \\
\hline Number of psychiatric hospitalizations, 1 year ago, mean [SD] & 0.61 & {$[0.9]$} & 0.7 & [0.9] & 0.5 & {$[0.8]$} & \multirow{2}{*}{0.147} \\
\hline $\mathrm{P}_{50}\left[\mathrm{P}_{25}-\mathrm{P}_{75}\right]$ & 0.0 & {$[0.0-0.1]$} & 1.0 & {$[0.0-1.0]$} & 0.0 & {$[0.0-1.0]$} & \\
\hline Number of drugs, mean [SD] & 3.4 & [1.9] & 3.5 & [1.9] & 3.3 & [1.9] & \multirow{2}{*}{0.638} \\
\hline $\mathrm{P}_{50}\left[\mathrm{P}_{25}-\mathrm{P}_{75}\right]$ & 3.0 & {$[2.0-4.0]$} & 3.0 & {$[2.0-4.0]$} & 3.0 & {$[2.0-5.0]$} & \\
\hline \multicolumn{8}{|l|}{ Treatment } \\
\hline Combination therapy & 61 & $(66.3)$ & 31 & $(63.3)$ & 30 & $(69.8)$ & 0.510 \\
\hline \multicolumn{8}{|l|}{ Therapeutic regimen } \\
\hline MS & 28 & $(30.4)$ & 17 & $(34.7)$ & 11 & $(25.6)$ & \multirow{5}{*}{0.481} \\
\hline MS + AAP/TYP & 27 & $(29.3)$ & 12 & $(24.5)$ & 15 & $(34.9)$ & \\
\hline MS + AAP/TYP + BZD/SA & 10 & $(10.9)$ & 4 & $(8.2)$ & 6 & $(13.9)$ & \\
\hline $\mathrm{MS}+\mathrm{AD}+\mathrm{BZD} / \mathrm{SA} / \mathrm{AAP}$ & 5 & $(5.4)$ & 2 & $(4.1)$ & 3 & $(7.0)$ & \\
\hline Other & 22 & $(24.0)$ & 14 & $(28.6)$ & 8 & $(18.6)$ & \\
\hline YMRS, mean [SD] & 2.4 & {$[3.7]$} & 2.2 & {$[3.2]$} & 2.7 & {$[4.1]$} & \multirow{2}{*}{0.956} \\
\hline $\mathrm{P}_{50}\left[\mathrm{P}_{25}-\mathrm{P}_{75}\right]$ & 0.0 & {$[0.0-4.0]$} & 1.0 & {$[0.0-4.0]$} & 0.0 & {$[0.0-5.0]$} & \\
\hline HDRS, mean [SD] & 2.0 & [2.9] & 2.1 & {$[3.2]$} & 1.9 & {$[2.5]$} & \multirow{2}{*}{0.911} \\
\hline $\mathrm{P}_{50}\left[\mathrm{P}_{25}-\mathrm{P}_{75}\right]$ & 1.0 & {$[0.0-3.0]$} & 1.0 & {$[0.0-3.0]$} & 1.0 & {$[0.0-3.0]$} & \\
\hline CGI, severity of illness, mean [SD] & 1.8 & {$[1.0]$} & 1.8 & {$[0.8]$} & 1.9 & {$[1.2]$} & 0.537 \\
\hline
\end{tabular}

to pharmacological and nonpharmacological interventions to achieve treatment goals.

\section{Outcome Data and Analysis}

Only the outcomes derived from the primary objective of the EMDADER-TAB study are reported in this article. ${ }^{21}$ The variables related to the primary outcomes were number of hospitalizations, emergency service consultations, unscheduled outpatient visits, and clinical evaluation of symptomatology.
Also included in this study were the uses of health services that were clearly related to psychiatric causes.

\section{Sample Size Calculation}

The sample size and power were calculated using EpiInfo version 6.04 (Centers for Disease Control and Prevention, Atlanta, GA). Assuming readmissions were $50 \%$ in the control group and $30 \%$ in the intervention group, with a confidence interval (CI) of $95 \%$ and a power of $80 \%$, it was necessary to include 


\section{Effectiveness of the Dader Method for Pharmaceutical Care on Patients with Bipolar I Disorder: Results from the EMDADER-TAB Study}

at least 166 patients. An estimated sample size of 200 patients with BD-I was needed to cover losses during follow-up and to increase sensitivity towards secondary objectives.

\section{Statistical Analysis}

For the analysis of the primary endpoint, the survival function by the nonparametric Kaplan-Meier method was used. ${ }^{22}$ To determine differences between the survival curves, the log-rank test was used, and the magnitude of differences between groups was assessed using the Cox model of proportional risk. Evaluation of clinical symptomatology was made through a general linear model for repeated measures by applying the Bonferroni correction factor and the intersubject factor (study group). The Young Mania Rating Scale and the Hamilton Depression Rating Scale (HDRS) were used to evaluate mania and depression. In addition to the assessment of disease severity through the Clinical Global Impression (CGI) scale, the ANCOVA test was used because the CGI scale refers to the longitudinal severity of illness during the year before the baseline evaluation.

\section{Ethical Approval}

Ethical approval for the study protocol was granted by the Faculty of Pharmaceutical Chemistry's Research Technical Committee at Universidad de Antioquia, the San Juan de Dios Clinic's Bioethics Committee (reference number 06/10) and 2 health care provider bioethics committees: COOMEVA (reference number 15/11) and SURA (reference number 3/11). Voluntary written informed consent was required from all participants.

\section{Results}

During the recruitment phase, the pharmacist reviewed 423 medical records of patients with a history of $\mathrm{BD}$, of which only 133 patients met the inclusion criteria. Forty-one patients refused to participate (Figure 2). Ninety-two patients were enrolled and randomized: 43 were assigned to the intervention group, and 49 were assigned to the control group. Of the 92 patients enrolled, 81 completed the study (38 in the intervention group and 43 in the control group). The mean [standard deviation] age of the patients was 42.8 [10.9] years, and 56.5\% of the population were female. The average drug prescribed was similar for both groups (3.4 [1.9]). The most frequent psychiatric treatment among the participating patients was the combination of atypical antipsychotics and mood stabilizers. The sociodemographic characteristics of the studied patients are shown in Table 1.

\section{Primary Outcomes}

Hospitalizations and Emergency Service Consultations. At 12 months of follow-up, only 1 hospitalization occurred in the intervention group that was associated with an adverse drug reaction, with a length of stay of 2 days. In contrast, the control group experienced more hospitalizations during the 12 months of follow-up (0-3 months: 5; 3-6 months: 3; 6-9 months: 1; 9-12 months: 7). The average length of stay was approximately 10 days (mean 9.9 [6.0]). The most frequent causes of hospitalization were depressive (47.1\%), manic (41.1\%), and mixed (5.9\%) episodes and adverse drug reactions (5.9\%).

The number of emergency service consultations were 23 (82.1\%) for the control groups versus 5 (17.9\%) for the intervention group. The strategies implemented by medical staff in the emergency department through the year were hospitalization (60.9\% control group vs. $0.0 \%$ intervention group), drug dose adjustment (21.7\% control group vs. $60.0 \%$ intervention group), and consultation with specialist (17.4\% control group vs. $40.0 \%$ intervention group).

The hospitalizations and emergency service consultations were significantly more frequent in the control group. Figure 3 illustrates 1-year Kaplan-Meier survival curves for psychiatric hospitalization and emergency service consultations, respectively, of EMDADER-TAB patients. These curves differed significantly for time to any recurrence: hospitalization (logrank $=8.09, P=0.004)$ and emergency service consultations (log-rank $=6.63, P<0.001)$.

Results of the Cox regression showed that age, gender, history of psychiatric hospitalization in the previous year, age of onset of illness, and social support did not affect the rate of hospitalizations and emergency service consultations during the 1-year follow-up period, with the exception of the study group variable. In both cases, only the study group variable was statistically significant $(P=0.042$ for hospitalizations and $P=0.034$ for emergency service consultations). Consequently, instantaneous risk of hospital admissions (hazard ratio $[\mathrm{HR}]=9.03,95 \% \mathrm{CI}=1.1-75.4)$ and emergency service consultations ( $\mathrm{HR}=3.38,95 \% \mathrm{CI}=1.1-10.5)$ were higher in the control group than in the intervention group, which is a result of the study intervention and not the influence of other variables.

Unscheduled Outpatient Visits. Unscheduled outpatient visits were significantly higher in the intervention group than in the control group $(P=0.023)$. In the intervention group, the reasons for an unscheduled outpatient visit were dose adjustment $(53.4 \%)$, administrative procedures $(20.0 \%)$, adverse drug reactions (13.3\%), and psychiatric assessment (13.3\%). In the control group, unscheduled outpatient visits were due to administrative procedures (1) and psychiatric assessment (2).

The 1-year Kaplan-Meier survival curve (Figure 4) showed that the incidence of unscheduled outpatient visits in patients receiving pharmaceutical care was higher than those patients receiving usual care, and the difference was statistically significant (log-rank $=5.87, P=0.015$ ). After adjusting the model for the same variables that were used for the evaluation of 
Effectiveness of the Dader Method for Pharmaceutical Care on Patients with Bipolar I Disorder:

Results from the EMDADER-TAB Study

\section{FIGURE 2 Patient Flowchart During the EMDADER-TAB Study}

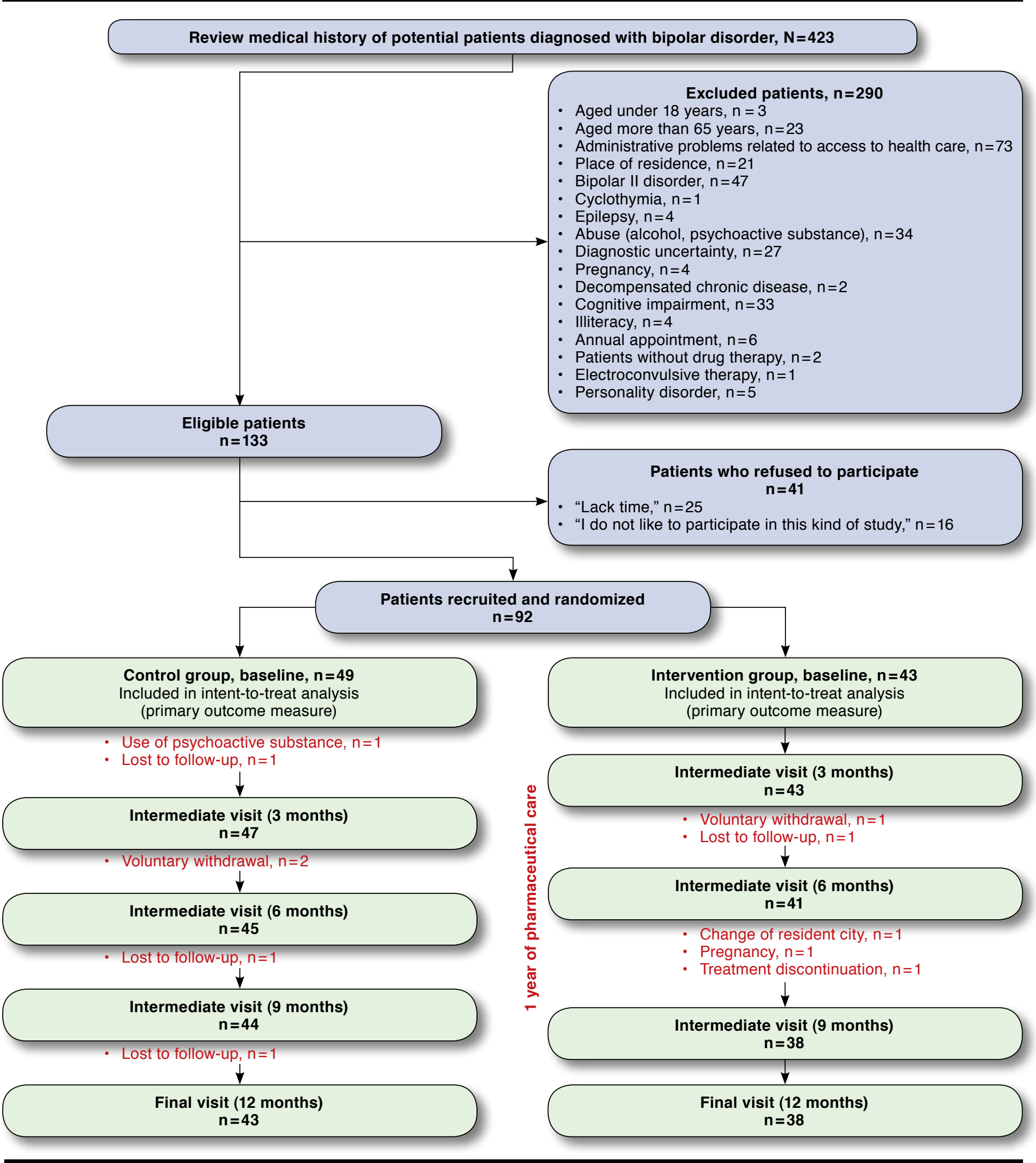




\section{A. Survival Curves (Hospitalization)}

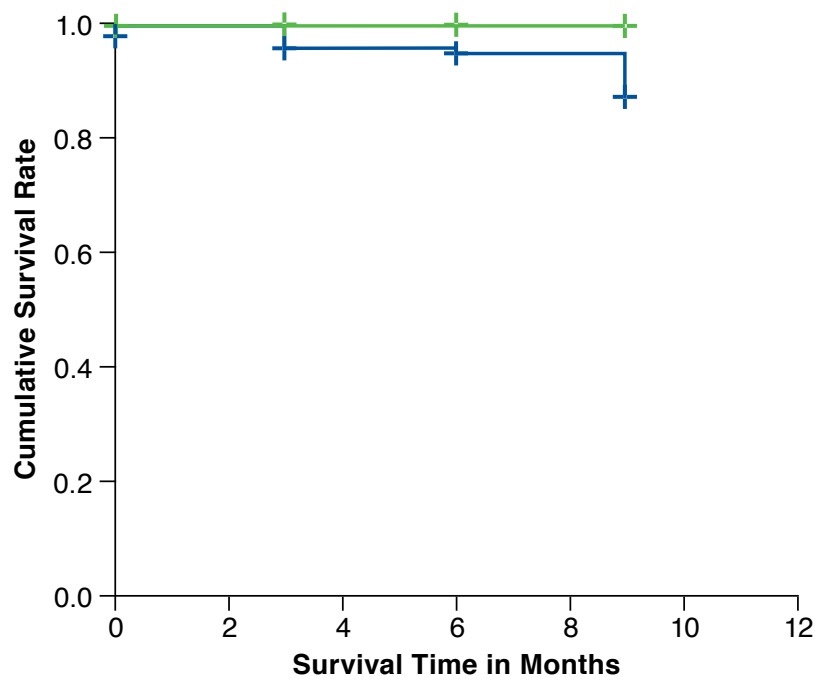

B. Survival Curves (Emergency Service Consultations)

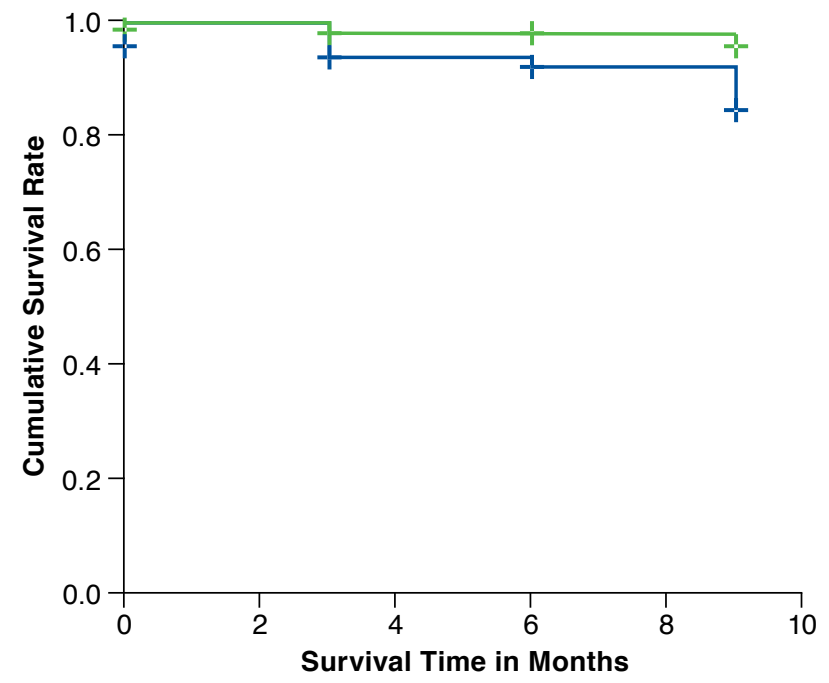

—Control group —-Intervention group +Control group-censored -Intervention group-censored

hospitalizations and emergency service consultations, only the study group variable was statistically significant $(H R=4.18$, $P=0.028$ ), meaning that the increase in the number of unscheduled outpatient visits in the intervention group is a result of receiving pharmaceutical care.

Clinical Evaluation of Depression and Mania. There was no significant group or group-time interaction effects for the HDRS. The between groups test indicated that the study group variable was significant $(F=3.224, P=0.013)$, and patients receiving pharmaceutical care showed greater statistically significant improvement in the HDRS compared with patients receiving usual care $(F=6.106, P=0.016)$. The evolution of depressive symptoms between groups showed that there was a significant decrease in the HDRS test scores in the intervention group (mean 0.88 [0.37]) compared with the control group (mean 2.15 [0.36]). Otherwise, the within subject test indicated that the groups did not change with regard to mania over time $(F=0.846, P=0.460)$; the between groups test indicated that the variable group was not significant $(P=0.671)$, that is, changes in symptomatology did not differ by treatment group in time.

Severity of Illness. There were significant group or grouptime interaction effects for the CGI, which showed that there is a significant time effect, and the groups did change in severity of disease over time $(P=0.049)$. Patients in the intervention group showed greater statistically significant improvement in the CGI compared with patients in the control group $(P=0.024)$. The ANCOVA test demonstrated that the intervention group (mean 1.3 [0.6]) had a significant increase in the improvement of disease severity than the control group, where there were no significant changes over time (mean 1.6 [ 0.7]). There was also a decrease in disease severity between the initial and final assessment. According to the Likert-type scale, the median score for the intervention group was 1 (normal), and the median score for the control group was 2 (minimum).

\section{Discussion}

The EMDADER-TAB trial was the first RCT conducted in Colombia that assessed the effect of a pharmaceutical intervention using the Dader Method on patients with BD-I measured by the decrease in the use of health services, except the unscheduled outpatient visits. The EMDADER-TAB trial also demonstrated the positive effect of using a pharmacist as part of a multidisciplinary mental health team in achieving therapeutic goals. Compared with usual care, pharmaceutical care had a statistically significant effect in reducing hospitalizations and emergency service consultations during a 1-year follow-up period, as well as an effect on the improvement of depressive symptoms and disease severity. The instantaneous risk of hospital admissions (HR $=9.03)$ and emergency service consultations (HR=3.38) was higher in the control group than the intervention group. 


\section{FIGURE 4 One-year Kaplan-Meier Survival Curves for EMDADER-TAB Patients: Unscheduled Outpatient Visits}

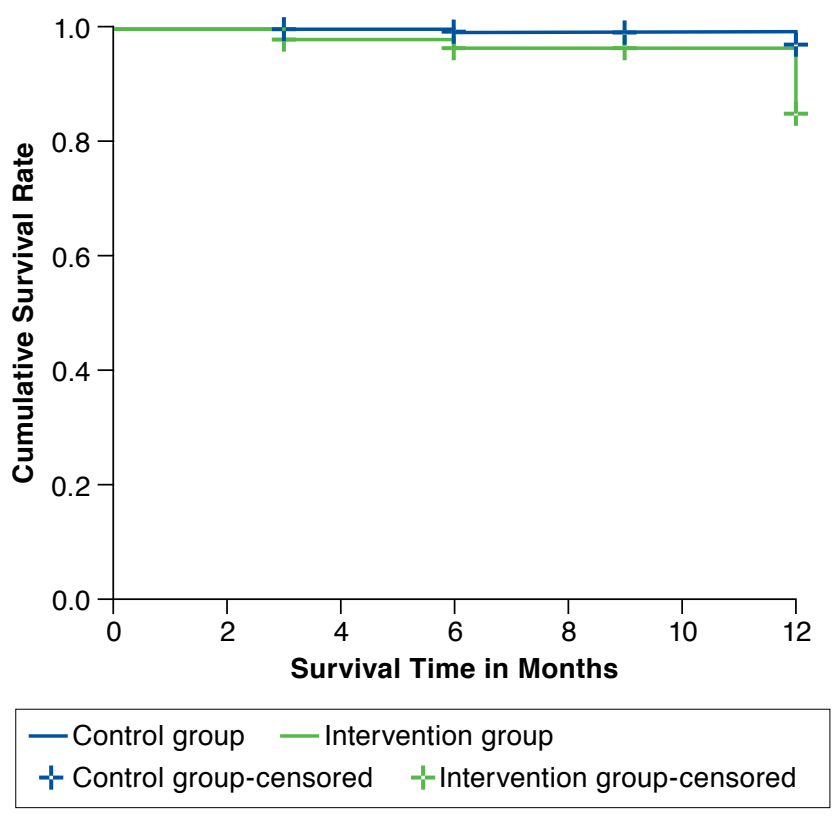

To our knowledge, there is a lack of RCTs designed to evaluate the effect of pharmaceutical interventions on the use of health services in patients with mental health disorders, which was the main objective of the EMDADER-TAB study. However, some studies have reported positive associations between pharmaceutical interventions and a decrease in hospitalizations and emergency service consultations, as well as an improvement in the outcomes of drug therapy in different health problems. ${ }^{23-25}$ Two recent studies conducted in Spain, ${ }^{26,27}$ showed that a community pharmacy-based medication review with a follow-up service delivered by a trained pharmacist had positive effects across clinical, economic, and humanistic outcomes in older adults (aged $>65$ years) with polypharmacy. However, the authors of these studies suggested that their findings be subject to a specific study designed and developed to generate more evidence to prove the cause and effect relationship between the use of medication review as a follow-up service (pharmaceutical care) and an improvement in the use of health services. To this end, the EMDADER-TAB results showed a cause and effect relationship between pharmaceutical care and the reduction of hospitalizations $(P=0.042)$ and emergency service consultations $(P=0.004)$ during a 1-year period - the only variable that was significantly related to this outcome was pharmaceutical care. Although the history of hospitalization during the 1 year of follow-up was associated with an increased risk of relapse, the correlation observed in this study was not statistically significant $(P=0.083)$.
In a study that evaluated the outcome of $\mathrm{BD}$ in the context of maintenance pharmacotherapy in 82 patients, $73 \%$ of the patients had a risk of manic or depressive relapse within 5 years, despite being in maintenance treatment. ${ }^{28}$ Moreover, two thirds of these patients had multiple relapses, and the number of episodes throughout life was associated with increased severity of symptoms. ${ }^{28}$ Since patients with BD-I have a high probability of hospitalization throughout life, the EMDADER-TAB results are significant in this regard. Patients who received pharmaceutical care were less likely to be hospitalized. Pharmaceutical care could be used as an intervention to identify possible risk factors that could precipitate a relapse.

An unexpected outcome from the EMDADER-TAB trial was the increase of unscheduled outpatient visits (4.18 times) by those patients receiving pharmaceutical care as compared with those patients receiving usual care. This outcome, however, not a negative one, rather it points to the strength of a pharmaceutical care intervention. For example, identification of prodromal symptoms by a patient prompts the patient to seek unscheduled assistance from the patient's mental health team. The pharmacist's role is highlighted in this process because of education provided by the pharmacist to the patient for the recognition and management of prodromal symptoms. ${ }^{29-31}$ The identification of prodromal symptoms and the seeking of timely assistance contributes to the prevention of hospitalizations, emergency service consultations, and clinical deterioration. As the evidence becomes stronger for the cause and effect relationship between pharmaceutical care and the prevention of hospitalizations and relapse care, hopefully there is a greater availability and flexibility for unscheduled outpatient visits, since it would be useless to educate patients in the identification of warning symptoms if they are limited to where they can find assistance. ${ }^{32}$

The results of this study indicate that pharmaceutical care, compared with usual care, promoted positive changes in depressive symptoms and severity of $\mathrm{BD}$. These findings are important because depressive symptoms are associated with an increased risk of suicide and functional impairment level. ${ }^{33}$ However, it is important to note that it was the pharmacist who carried out the evaluation of depressive symptomatology using the HDRS, which could constitute a bias in the results. Furthermore, depressive symptoms are more frequent than mania during the course of BD. ${ }^{34}$ Similarly, it has been observed that patients with BD-I have more depressive periods (67.0\%) than manic periods (20.0\%), with $13.0 \%$ having mixed dominance. ${ }^{35}$

The EMDADER-TAB results show that despite different measurements in clinical symptomatology, the leading causes of hospitalization were related to depressive (47.1\%) and manic (41.1\%) episodes, which could explain why no significant changes were observed between different measures of the 


\section{Effectiveness of the Dader Method for Pharmaceutical Care on Patients with Bipolar I Disorder: Results from the EMDADER-TAB Study}

HDRS for depression over time in both groups, but yet the significant effect of the reduction of the HDRS score (mean 0.88 [0.37]) was in the intervention group rather than the control group (mean 2.15 [0.36]). Moreover, it was observed that pharmaceutical care had no significant effect on manic symptoms, which differs from what is found in the literature, where it has been shown that prodromal symptoms of mania are more easily identifiable than depressive prodromes, ${ }^{36}$ since manic symptoms are behavioral, whereas depressive symptoms are cognitive and affective. ${ }^{37}$ Another explanation could be the presence of milder symptoms, which might be more difficult to identify during a clinical intervention. However, interpretations of these results exceed the objectives of this work.

In the longitudinal evaluation of illness severity, there was symptomatic improvement in both groups, but the intervention group experienced significantly higher improvement. This result could be associated with less severe and residual symptoms in this group, which resulted in a decrease in relapses and hospitalizations. ${ }^{38}$ In addition, weekly monitoring of patients in the intervention group allowed for more frequent identification of residual symptoms, which resulted in more timely intervention, compared with the control group where evaluations did not have the same frequency, so identification of acute symptoms that might trigger a relapse or hospitalization became more difficult.

\section{Limitations}

There are limitations to this study that need to be considered. First, the study results might have been subject to potential bias because there was no placebo treatment; the study patients were not blinded to treatment assignment; and both groups were seen by the same pharmacist. However, potential bias was reduced by use of a standardized process for measurement in both groups. Patients in the control group were also provided with written and oral education about BD, mental health, and drugs and were counseled concerning therapeutic goals and the importance of adherence to pharmacological and nonpharmacological interventions to achieve those goals. The effect of written educational material received by the patients in the control group might have produced positive outcomes and reduced the magnitude of differences compared with the intervention group.

Second, exclusion criteria could have limited the participation of more patients who could have benefited from the intervention. The final sample size (92 patients) was smaller than expected (166 patients). However, in order to avoid a noticeable variation in recruitment time and not to significantly modify the internal validity of the study, it was decided to finish the enrollment of patients after 18 months of recruitment because the sample was representative to respond to the primary objective of the test. In addition, there was a recalculation of the sample size at final recruitment time. Thus, we assumed for the sample calculation an incidence ratio between groups of $50 \%$. But at the final recruitment time, we observed a ratio of $10 \%$ for the primary variable. With a significance level of 0.05 (2-tailed) and a power of $80 \%$, we estimated a required sample of 84 patients (42 in each group). Consequently, a final sample size of 92 patients was considered appropriate for the primary outcome of the EMDADER-TAB study.

Third, certain variables, such as the number of episodes and hospitalizations throughout life, predominant polarity, and insight were not assessed; however, they should be considered for further studies. Moreover, a pharmacist trained in pharmaceutical care using the Dader Method performed the evaluations in the EMDADER-TAB study, so caution should be exercised in interpreting the results more broadly. Finally, it should be noted that this study's results are limited to BD-I patients, which limits the degree to which the present study findings can be generalized to other settings. However, this study can be considered as the beginning of future research in different fields of mental health.

\section{Conclusions}

Compared with usual care, complemented with verbal and written information about the disease and drugs, pharmaceutical care, based on the Dader Method, significantly reduced hospitalizations and emergency service consultations for patients with BD-I. The EMDADER-TAB trial demonstrated the positive effect of pharmaceutical care on treated BD-I patients compared with usual care.

\section{Authors}

ANDREA SALAZAR-OSPINA, PhD, PharmD; PEDRO AMARILES, PhD, PharmD; and JAIME A. HINCAPIÉ-GARCÍA, BPharm, MSc, Grupo Promoción y Prevención Farmacéutica, Facultad de Ciencias Farmacéuticas y Alimentarias, Universidad de Antioquia UdeA, Medellin, Colombia. SEBASTIÁN GONZÁLEZ-AVENDAÑO, MD, Grupo Promoción y Prevención Farmacéutica, Facultad de Ciencias Farmacéuticas y Alimentarias, Universidad de Antioquia UdeA, Medellin, Colombia, and Humax Pharmaceutical, La Estrella, Antioquia, Colombia. DORA M. BENJUMEA, PhD, PharmD, Programa de Ofidismo y Escorpionismo, Facultad de Ciencias Farmacéuticas y Alimentarias, Universidad de Antioquia UdeA, Medellín, Colombia; MARIA JOSÉ FAUS, PhD, PharmD, Facultad de Farmacia, Universidad de Granada, Granada, Spain; and LUIS F. RODRIGUEZ, MD, Orden Hospitalaria San Juan de Dios, Clinica San Juan de Dios, La Ceja, Antioquia, Colombia.

AUTHOR CORRESPONDENCE: Andrea Salazar-Ospina, PhD, PharmD, Grupo Promoción y Prevención Farmacéutica, Facultad de Ciencias Farmacéuticas y Alimentarias, Universidad de Antioquia UdeA, Calle 70 No 52-21, Medellin, Colombia. Tel.: +574.2195460; Fax: +574.2195456; E-mail: andrea.salazaro@udea.edu.co. 


\section{Effectiveness of the Dader Method for Pharmaceutical Care on Patients with Bipolar I Disorder: Results from the EMDADER-TAB Study}

\section{DISCLOSURES}

This study received funding from the Universidad de Antioquia, Committee for Development Research and Sustainability Program, CODI, (2013-2014 and 2014-2015). Humax Pharmaceutical provided support for the initial development of the EMDADER-TAB trial without commercial interest in the outcomes derived from the trial. Salazar-Ospina reports grants from Credito Beca Francisco José de Caldas Scholarship for Doctoral Programs (528), which also contributed to the support of this study. González-Avendaño is an employee of Humax Pharmaceutical. The other authors have nothing to disclose.

Study concept and design were contributed by Benjumea, Faus, and Rodriguez, along with Salazar-Ospina and Amariles. Salazar-Ospina took the lead in data collection, assisted by González-Avendaño, and data interpretation was performed by Salazar-Ospina, Hincapié-García, and GonzálezAvendaño. The manuscript was written primarily by Salazar-Ospina, with assistance from Amariles and González-Avendaño, and revised by all the authors.

\section{REFERENCES}

1. American Psychiatric Association. Diagnostic and Statistical Manual of Mental Disorders, Fifth Edition (DSM-5). Arlington, VA: American Psychiatric Publishing; 2013.

2. Jamison KR. Suicide and bipolar disorder. J Clin Psychiatry. 2000;61(Suppl 9):47-51.

3. Brenner CJ, Shyn SI. Diagnosis and management of bipolar disorder in primary care: a DSM-5 update. Med Clin North Am. 2014;98(5):1025-48. Available at: http://www.medical.theclinics.com/article/S0025-

7125(14)00092-3/fulltext. Accessed November 28, 2016.

4. Ministerio de la Protección Social and Fundación FES Social. Estudio Nacional de Salud Mental-Colombia, 2003 [National Mental Health Study-Colombia, 2003]. 2005. Available at: https://www.minsalud.gov.co/Documentos\%20y $\% 20$ Publicaciones/ESTUDIO\%20NACIONAL\%20DE\%20SALUD\%20MENTAL\%20 EN\%20COLOMBIA.pdf. Accessed November 28, 2016.

5. Velligan DI, Weiden PJ, Sajatovic M, et al. The expert consensus guideline series: adherence problems in patients with serious and persistent mental illness. J Clin Psychiatry. 2009;70(Suppl 4):1-46.

6. Kessler RC, Chiu WT, Demler O, Merikangas KR, Walters EE. Prevalence, severity, and comorbidity of 12-month DSM-IV disorders in the National Comorbidity Survey Replication. Arch Gen Psychiatry. 2005;62(6):617-27. Available at: http://jamanetwork.com/journals/jamapsychiatry/fullarticle/ 208671. Accessed November 28, 2016

7. Guo JJ, Keck PE, Li H, Jang R, Kelton CML. Treatment costs and health care utilization for patients with bipolar disorder in a large managed care population. Value Health. 2008;11(3):416-23. Available at: http://onlinelibrary.wiley.com/doi/10.1111/j.1524-4733.2007.00287.x/abstract. Accessed November 28, 2016.

8. Ohsfeldt RL, Lage MJ, Rajagopalan K. Medication use, service utilization, and medical costs associated with new episodes of bipolar disorder: evidence from a retrospective claims database. Prim Care Companion J Clin Psychiatry. 2007;9(4):280-86.

9. Finley PR, Crismon ML, Rush AJ. Evaluating the impact of pharmacists in mental health: a systematic review. Pharmacotherapy. 2003;23(12):1634-44.

10. Bell S, McLachlan AJ, Aslani P, Whitehead P, Chen TF. Community pharmacy services to optimise the use of medications for mental illness: a systematic review. Aust New Zealand Health Policy. 2005;2:29. Available at: https://www.ncbi.nlm.nih.gov/pmc/articles/PMC1345690/. Accessed November 28, 2016

11. Bell JS, Rosen A, Aslani P, Whitehead P, Chen TF. Developing the role of pharmacists as members of community mental health teams: perspectives of pharmacists and mental health professionals. Res Social Adm Pharm. 2007:3(4):392-409.
12. Ilickovic IM, Jankovic SM, Tomcuk A, Djedovic J. Pharmaceutical care in a long-stay psychiatric hospital. Eur J Hosp Pharm. Published online November 27, 2015

13. Marques LAM, Galduróz JCF, Fernandes MR, Oliveira CCO, Beijo LA, Noto AR. Assessment of the effectiveness of pharmacotherapy follow-up in patients treated for depression. J Manag Care Pharm. 2013;19(3):218-27. Available at: http://www.jmcp.org/doi/10.18553/jmcp.2013.19.3.218.

14. Faus Dáder MJ, Amariles Muñoz P, Martínez Martínez F. Atención Farmacéutica: Conceptos, Procesos Y Casos Prácticos. Madrid: Ergón; 2008.

15. Alós M, Álvarez de-Toledo F, Ausejo M, et al. Foro de Atención Farmacéutica. Doumento de Consenso. 2008. Available at: http://www. pharmaceutical-care.org/archivos/804/foro_at_farma.pdf. Accessed November 28, 2016

16. Pharmaceutical Care Research Group, University of Granada (Spain). Dader method to provide pharmacotherapy follow up. Ars Pharm. 2005;46(4):309-37.

17. Pharmaceutical Care Research Group, University of Granada (Spain). Pharmacotherapy follow-up: The Dader method (3rd revision: 2005). Pharm Pract (Granada). 2006;4(1):44-53

18. Sabater Hernández D, Silva Castro MM FM. Dader Method: Guidelines for Pharmacotherapy Follow-up. 3rd ed. Granada, Spain: Pharmaceutical Care Research Group, University of Granada; 2007.

19. Sabater D. Tipos de intervenciones farmacéuticas en seguimiento farmacoterapéutico [Types of pharmacist intervention in pharmacotherapy followup]. Farmacoter. 2005;3(2):90-97.

20. Rubio-Valera M, Chen TF, O'Reilly CL. New roles for pharmacists in community mental health care: a narrative review. Int J Environ Res Public Health. 2014;11(10):10967-90. Available at: https://www.ncbi.nlm.nih.gov/ pmc/articles/PMC4211017/. Accessed November 28, 2016.

21. Salazar-Ospina A, Amariles P, Benjumea DM, Gutierrez F, Faus MJ, Rodriguez LF. Effectiveness of the Dader Method for pharmaceutical care in patients with bipolar I disorder: EMDADER-TAB: study protocol for a randomized controlled trial. Trials. 2014;15(1):174.

22. Arribalzaga EB. Interpretación de las curvas de supervivencia. Rev Chil cirugía. 2007;59(1):75-83. Available at: http://www.scielo.cl/scielo. php?script=sci_arttext $\&$ pid=S0718-40262007000100013. Accessed November 28, 2016

23. Tommelein E, Mehuys E, Van Hees T, et al. [Effectiveness of pharmaceutical care for patients with COPD: translated review of the recently published PHARMACOP triall. J Pharm Belg. 2014;(3):4-14 [Article in French].

24. Wei L, Yang X, Li J, et al. Effect of pharmaceutical care on medication adherence and hospital admission in patients with chronic obstructive pulmonary disease (COPD): a randomized controlled study. J Thorac Dis. 2014;6(6):656-62. Available at: https://www.ncbi.nlm.nih.gov/pmc/articles/ PMC4073386/. Accessed November 28, 2016.

25. Sadik A, Yousif M, McElnay JC. Pharmaceutical care of patients with heart failure. Br J Clin Pharmacol. 2005;60(2):183-93. Available at: https://www.ncbi. nlm.nih.gov/pmc/articles/PMC1884928/. Accessed November 28, 2016.

26. Martinez-Martinez F, Farragher T, Faus-Dader M, et al. Measurement of the Clinical, Economic and Humanistic Impact of the Pharmacotherapy Follow-Up Service in Polymedicated Elderly in the Spanish Community Pharmacy. conSIGUE. Report 2011-2014. Madrid: General Council of Associations of Pharmacists; 2014 [in Spanish].

27. Ocampo CC, Garcia-Cardenas V, Martinez-Martinez F, Benrimoj SI, Amariles P, Gastelurrutia MA. Implementation of medication review with follow-up in a Spanish community pharmacy and its achieved outcomes. Int J Clin Pharm. 2015;37(5):931-40.

28. Gitlin MJ, Swendsen J, Heller TL, Hammen C. Relapse and impairment in bipolar disorder. Am J Psychiatry. 1995;152(11):1635-40. 


\section{Effectiveness of the Dader Method for Pharmaceutical Care on Patients with Bipolar I Disorder: \\ Results from the EMDADER-TAB Study}

29. Perry A, Tarrier N, Morriss R, McCarthy E, Limb K. Randomised controlled trial of efficacy of teaching patients with bipolar disorder to identify early symptoms of relapse and obtain treatment. BMJ. 1999;318(7177):149-53.

30. Chisholm-Burns MA, Kim Lee J, Spivey CA, et al. US pharmacists' effect as team members on patient care: systematic review and meta-analyses. Med Care. 2010;48(10):923-33. Available at: http://journals.lww.com/lww-medicalcare/pages/articleviewer.aspx?year $=2010$ \&issue $=10000 \&$ article $=00010 \& t$ ype $=$ abstract. Accessed November 28, 2016.

31. Lin HC, Lee HC. The association between timely outpatient visits and the likelihood of rehospitalization for schizophrenia patients. Am J Orthopsychiatry. 2008;78(4):494-97.

32. Colom F. Keeping therapies simple: psychoeducation in the prevention of relapse in affective disorders. Br J Psychiatry. 2011;198(5):338-40. Available at: http://bjp.rcpsych.org/content/198/5/338. Accessed November 28, 2016

33. Post RM. The impact of bipolar depression. J Clin Psychiatry. 2005;66(Suppl 5):5-10.
34. Judd LL, Akiskal HS, Schettler PJ, et al. The long-term natural history of the weekly symptomatic status of bipolar I disorder. Arch Gen Psychiatry. 2002;59(6):530-37.

35. Kurtz MM, Gerraty RT. A meta-analytic investigation of neurocognitive deficits in bipolar illness: profile and effects of clinical state. Neuropsychology. 2009;23(5):551-62. Available at: https://www.ncbi.nlm.nih.gov/pmc/articles/ PMC2804472/. Accessed November 28, 2016.

36. Jackson A, Cavanagh J, Scott J. A systematic review of manic and depressive prodromes. J Affect Disord. 2003;74(3):209-17.

37. Sierra P, Livianos L, Arques S, Castelló J, Rojo L. Prodromal symptoms to relapse in bipolar disorder. Aust N Z J Psychiatry. 2007;41(5):385-91.

38. Perlis RH, Ostacher MJ, Patel JK, et al. Predictors of recurrence in bipolar disorder: primary outcomes from the Systematic Treatment Enhancement Program for Bipolar Disorder (STEP-BD). Am J Psychiatry. 2006;163(2):217-24. Available at: http://ajp.psychiatryonline.org/doi/full/10.1176/appi.ajp.163.2.217. Accessed November 28, 2016. 